\title{
Aeronautical and Aerospace Material and Structural Damages to Failures: Theoretical Concepts
}

\author{
Andriy Viktorovich Goncharenko \\ Aircraft Airworthiness Retaining Department, Educational and Research Airspace Institute, National Aviation University, 1 \\ Kosmonavta Komarova Avenue, Kyiv 03058, Ukraine
}

Correspondence should be addressed to Andriy Viktorovich Goncharenko; andygoncharenco@yahoo.com

Received 29 December 2017; Revised 5 March 2018; Accepted 1 April 2018; Published 22 April 2018

Academic Editor: Paulo M. S. T. de Castro

Copyright (C) 2018 Andriy Viktorovich Goncharenko. This is an open access article distributed under the Creative Commons Attribution License, which permits unrestricted use, distribution, and reproduction in any medium, provided the original work is properly cited.

\begin{abstract}
The goal of this paper is to investigate the possible directions of some specified methods for aeronautical and aerospace material and structure effectiveness modeling and optimization. Multioptionality hybrid function uncertainty conditional optimization doctrine application is supposed to be implemented for a degrading failure problem optimal solution determination. The optimal solution is assumed to deliver the maximum value to the probability of damage but not the failure state of the studied material behavior. The principal supposition is that there should be some certain objectively existing value extremized in the conditions of the hybrid optional function uncertainty. There is a scientific proof for the choice of a good maintenance optimal periodicity method that fits the customer's needs, taking into account the effectiveness functions pertaining to the options. The described doctrine allows obtaining the objectively existing optimal values not with the help of a probabilistic but rather with a multioptimal concept. The subjective entropy maximum principle is the other paradigm concept involved in the considered problem solution, which is an equivalent for the uncertainty conditional optimization at the optimal hybrid function distribution determination. By applying simplified, however possible, models and expressions for effectiveness, plausible results are obtained and illustrated in diagrams visualizing the situation and allowing for the selection of a good choice. The ideas of the required material method choice optimization with respect to only two simple parameters, nevertheless, develop numerous particular combinations. Moreover, an increase in the number of parameters and further complication of the problem setting will not change the principle of the problem solution.
\end{abstract}

\section{Introduction}

The complex process of choosing materials for the airspace industry commonly follows a systematic approach based on multiple requirements and specifications and supported by extensive studies. The executors' individual preferences are an essential factor at this process. It is also important to note that the selection and use of one or another type of material for aerospace use, development of aeronautical engineering and aircraft element structural design, and contemplation of the appropriate fabrication methods are a matter of someone's choice. Therefore, ensuring maximum reliability and efficiency in the field of aeronautics and aerospace, while controlling related costs, faces great pressure for ever increasing performance. Thus, the problem of optimization plays a central role among many different contributions for achieving those objectives and goes far beyond the framework of basic engineering optimization.

The theory of subjective analysis [1] deals with the scientific explanations of the human's behavior in situations of multialternativeness uncertainty. The theory is developed especially for an evaluation of the individuals' subjective preferences. The preference functions in their turn are found in an explicit view. Moreover, the distribution of the preference functions is obtained with the help of an optimization on the basis of an entropy paradigm. The key point is the so-called subjective entropy maximum principle (SEMP). The core of SEMP is the statement postulating the optimality 


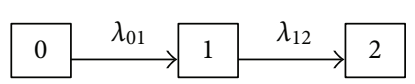

FIGURE 1: Graph of the states of a system with a degrading failure.

of the individual's subjective preference distribution with regard to the achievable alternative uncertainty degree measured by subjective entropy [1, Section 3.5, pp. 141-149].

The doctrine proposed in this paper uses a similar mathematical apparatus; however, an original objective [2-4] versus subjective theoretical concept is described, applied, and discussed in $[1,5]$ for finding certain extremal values of reliability measures (probabilities). In those cases, studied in the works of $[1,5]$, the use of SEMP is essential for optimal decision making.

Possible fields of the proposed approach application are morphing aeronautical structures $[6,7]$, different investigations on the influence of process parameters [8], and effects of heat treatment on fatigue [9] crack growth. Also, the models can be used at modeling the volume-based strain energy density approach [10], as well as fatigue strength subject to cyclic loading [11] and modifications of the activation energy, like for the material purposes discussed in the Sixth World Congress report in [12].

The presented paper's theoretical concept is centered upon material degradation issues.

\section{Mathematical Modeling and Developed Methods}

2.1. Multioptionality Hybrid Function Uncertainty Conditional Optimization Doctrine Application. In previous publications [2-4], a multioptional way for determining the optimal solution for a degrading failure problem was shown.

It is proposed to consider modeling and optimizing structural behavior and damage of advanced materials for aerospace use with the help of a graph presented in Figure 1.

In Figure 1, "0" denotes the up state of the system, "1" denotes damage, and " 2 " denotes failure. The corresponding system state transition intensities are depicted as $\lambda_{01}$ and $\lambda_{12}$.

The situation portrayed with the graph illustrated in Figure 1 is a simplified case for calculating the probabilities of the relating states " 0 ", "1", and "2": $P_{0}, P_{1}$, and $P_{2}$, developing in time $t$.

In order to determine the "0", " 1 ", and "2" states' probabilities, it is proposed to implement the specially introduced works in [2-4] on hybrid optional-probabilistic approach function distribution entropy conditional optimization doctrine.

The principal supposition is that there should be some certain objectively existing value extremized in conditions of the hybrid optional function uncertainty. This is a radically different ideology from that of subjective analysis $[1,5]$, where it is postulated that an individual's subjective optimal distribution of her/his preference functions accordingly with the achievable set of alternatives that she/he has taken into consideration, that is, SEMP.

With the use of the supposed multioptional optimality, likewise in subjective analysis $[1,5]$ conditional optimality of the individual's subjective preference distribution with extremizing subjective entropy, that is, applying the doctrine analogous to the SEMP concept, we have the right to write down the postulated functional in view of $[2-4]$ :

$$
\begin{aligned}
\Phi_{h}= & -\sum_{i=1}^{2} h_{i}\left(F_{i}, x\right) \ln h_{i}\left(F_{i}, x\right)+t_{p}^{*} \sum_{i=1}^{2} h_{i}\left(F_{i}, x\right) F_{i}(\cdot) \\
& +\gamma\left[\sum_{i=1}^{2} h_{i}\left(F_{i}, x\right)-1\right],
\end{aligned}
$$

where $h_{i}\left(F_{i}\right)$ are specific hybrid optional effectiveness functions of the option effectiveness functions: $F_{i}$ and $h_{i}\left(F_{i}\right)$ are similar to the preference functions of $[1,5]$, however, in this problem setting the assumed specific hybrid optional effectiveness functions $h_{i}\left(F_{i}\right)$ are not related with anybody's preferences or choice; $x$ is unknown, an uncertain multiplier of the Lagrange type; $t_{p}{ }^{*}$ is the system's (see Figure 1) and the system's described process intrinsic parameter, suspected to be an optimal (in such a problem setting, $t_{p}{ }^{*}$, which is accepted by an assumption optimal value of maintenance periodicity, is going to deliver the maximal value to the probability $P_{1}(t)$ of the damaged but still not fractured (failure) state "1" of a material, [2-4]); $\gamma$ is the normalizing coefficient (function).

The solution $t_{p}{ }^{*}$ of the objective functional in (1) is obtained on the basis of the necessary conditions for the extremum existence of the functional in (1):

$$
\frac{\partial \Phi_{h}}{\partial h_{i}\left(F_{i}, x\right)}=0 .
$$

The condition of (2) yields

$$
\frac{\partial \Phi_{h}}{\partial h_{i}(\cdot)}=-\ln h_{i}(\cdot)-1+t_{p}^{*} F_{i}(\cdot)+\gamma=0, \quad \forall i=\overline{1,2}
$$

This inevitably means in turn

$$
\ln h_{1}(\cdot)-t_{p}^{*} F_{1}(\cdot)=\gamma-1=\ln h_{2}(\cdot)-t_{p}^{*} F_{2}(\cdot) .
$$

From where

$$
\ln h_{1}(\cdot)-\ln h_{2}(\cdot)=t_{p}^{*}\left[F_{1}(\cdot)-F_{2}(\cdot)\right] .
$$

And in analogous way to (2),

$$
t_{p}^{*}=\frac{\ln h_{1}(\cdot)-\ln h_{2}(\cdot)}{F_{1}(\cdot)-F_{2}(\cdot)} .
$$

The two considered optional processes (see Figure 1) are as follows:

(i) Option number 1 is to go out from state "0" or to come into state "1" (in the presented circumstances, both are the same).

(ii) Option number 2 is to go out from state " 1 " or to come into state " 2 " (in the presented circumstances, both are the same). 
Hence, it is obvious that the effectiveness functions relating with the options are supposed as follows:

$$
F_{1}=\lambda_{01}
$$

For the second option,

$$
F_{2}=\lambda_{12} \text {. }
$$

The corresponding hybrid optional effectiveness function would be

$$
\begin{aligned}
& h_{1}=x F_{1}=x \lambda_{01}, \\
& h_{2}=x F_{2}=x \lambda_{12} .
\end{aligned}
$$

Substituting the relevant interrelationships of (7), (8), (9), and (10) for their values into the sought expression in (6) of the optimal periodicity $t_{p}{ }^{*}$, one obtains

$$
t_{p}^{*}=\frac{\ln \left(x \lambda_{01}\right)-\ln \left(x \lambda_{12}\right)}{\lambda_{01}-\lambda_{12}}=\frac{\ln \left(x \lambda_{01} / x \lambda_{12}\right)}{\lambda_{01}-\lambda_{12}} .
$$

Finally, (11) yields

$$
t_{p}^{*}=\frac{\ln \left(\lambda_{01}\right)-\ln \left(\lambda_{12}\right)}{\lambda_{01}-\lambda_{12}} .
$$

One has to emphasize here that optimal periodicity $t_{p}{ }^{*}$ in view of (12) and also obtained in [2] has been found not in a probabilistic way but rather in an optimal multioptional way.

The sense of the uncertain multiplier $x$ becomes obvious with the use of the normalizing condition of the initial functional in (1). That is, with the use of the presupposed relations of (7), (8), (9), and (10)

$$
\sum_{i=1}^{2} h_{i}\left(F_{i}, x\right)=1=\left(h_{1}=x \lambda_{01}\right)+\left(h_{2}=x \lambda_{12}\right),
$$

which yields

$$
x=\frac{1}{\lambda_{01}+\lambda_{12}} .
$$

Therefore, from the expressions for the corresponding hybrid optional effectiveness functions in (9) and (10) it follows that

$$
h_{1}=\frac{\lambda_{01}}{\lambda_{01}+\lambda_{12}} \text {. }
$$

Hence,

$$
h_{2}=\frac{\lambda_{12}}{\lambda_{01}+\lambda_{12}} \text {. }
$$

2.2. SEMP Paradigm Concept Equivalent Uncertainty Conditional Optimization Hybrid Function Distribution Determination. Concerning the objective functional in (1), it is possible to find an optimal distribution of the hybrid optional effectiveness functions starting with the functional (1) extremum existence conditions of (2) and (3).
This gives

$$
\ln h_{i}(\cdot)=\gamma-1+t F_{i}(\cdot) .
$$

Here in (17), the parameter of time $t$ is implemented. It is put into the objective functional in (1) instead of $t_{p}{ }^{*}$. This was done because time now is deemed to be an independent variable. Therefore, this parameter has to be reckoned with as the variable to be optimized.

Then from (17),

$$
h_{i}(\cdot)=\exp \left[\gamma-1+t F_{i}(\cdot)\right]
$$

The normalizing condition application, likewise expressed with (13), yields

$h_{1}(\cdot)+h_{2}(\cdot)=1=\exp (\gamma-1)\left\{\exp \left[t F_{1}(\cdot)\right]+\exp \left[t F_{2}(\cdot)\right]\right\}$.

From where

$$
\exp (\gamma-1)=\frac{1}{\exp \left[t F_{1}(\cdot)\right]+\exp \left[t F_{2}(\cdot)\right]}
$$

At last,

$$
h_{i}^{o p t}(\cdot)=\frac{\exp \left[t F_{i}(\cdot)\right]}{\sum_{j=1}^{2} \exp \left[t F_{j}(\cdot)\right]} .
$$

The obtained optimal distribution, described with (21) of the hybrid optional functions, ensures the conditional extremum (maximum) for the hybrid optional function entropy or the maximum to the objective functional in (1).

Indeed, one can easily make sure that the extremum existence conditions of the second order, from (3), are as follows:

$$
\frac{\partial^{2} \Phi_{h}}{\partial h_{i}\left(F_{i}, x\right)^{2}}=-\frac{1}{h_{i}\left(F_{i}, x\right)}<0 .
$$

This inequality of (22) is satisfied at any "point", since the hybrid optional functions always have positive values ] $0 . .1$ [ in the postulated view functional for multioptional consideration, like (1), including the "point" suspected for the extremum on the condition of (2).

Moreover, although the optimally distributed hybrid optional functions expressed with (21) are different from the hybrid optional functions described with (9) and (10), as well as their developed transformed versions in the view of (15) and (16), in the case of (6), (7), (8), (9), (10), (11), (12), (13), (14), (15), and (16), the functions of (21) and (15) and (16) have the same value.

2.3. An Idea of Combining the Concepts. It can be easily seen that if the optimal periodicity $t_{p}{ }^{*}$, in view of (12), is substituted for the value of time into the optimal distribution of hybrid optional functions represented by (21), then (15) and (16) will be derived. 
Indeed,

$$
\left.h_{1}^{\mathrm{opt}}(\cdot)\right|_{t=t_{p}^{*}}=\frac{\exp \left[t_{p}^{*} F_{1}(\cdot)\right]}{\exp \left[t_{p}^{*} F_{1}(\cdot)\right]+\exp \left[t_{p}^{*} F_{2}(\cdot)\right]} .
$$

Then,

$$
\exp \left[t_{p}^{*} F_{1}(\cdot)\right]=\exp \left[\frac{\ln \left(\lambda_{01}\right)-\ln \left(\lambda_{12}\right)}{\lambda_{01}-\lambda_{12}} \lambda_{01}\right] .
$$

And equally,

$$
\begin{aligned}
& \exp \left[t_{p}^{*} F_{2}(\cdot)\right]=\exp \left[\frac{\ln \left(\lambda_{01}\right)-\ln \left(\lambda_{12}\right)}{\lambda_{01}-\lambda_{12}} \lambda_{12}\right], \\
& \exp \left[t_{p}^{*} F_{1}(\cdot)\right]=\exp \left[\ln \left(\frac{\lambda_{01}}{\lambda_{12}}\right)\right]^{\lambda_{01} /\left(\lambda_{01}-\lambda_{12}\right)}, \\
& \exp \left[t_{p}^{*} F_{2}(\cdot)\right]=\exp \left[\ln \left(\frac{\lambda_{01}}{\lambda_{12}}\right)\right]^{\lambda_{12} /\left(\lambda_{01}-\lambda_{12}\right)}, \\
& \exp \left[t_{p}^{*} F_{1}(\cdot)\right]=\left(\frac{\lambda_{01}}{\lambda_{12}}\right)^{\lambda_{01} /\left(\lambda_{01}-\lambda_{12}\right)}, \\
& \exp \left[t_{p}^{*} F_{2}(\cdot)\right]=\left(\frac{\lambda_{01}}{\lambda_{12}}\right)^{\lambda_{12} /\left(\lambda_{01}-\lambda_{12}\right)} .
\end{aligned}
$$

From (23),

$$
\left.h_{1}^{\mathrm{opt}}(\cdot)\right|_{t=t_{p}^{*}}=\frac{1}{1+\left(\exp \left[t_{p}^{*} F_{2}(\cdot)\right] / \exp \left[t_{p}^{*} F_{1}(\cdot)\right]\right)} .
$$

Therefore,

$$
\left.h_{1}^{\mathrm{opt}}(\cdot)\right|_{t=t_{p}^{*}}=\frac{1}{1+\left(\left(\lambda_{01} / \lambda_{12}\right)^{\lambda_{12} /\left(\lambda_{01}-\lambda_{12}\right)} /\left(\lambda_{01} / \lambda_{12}\right)^{\lambda_{01} /\left(\lambda_{01}-\lambda_{12}\right)}\right)},
$$

$$
\begin{aligned}
& \left.h_{1}^{\mathrm{opt}}(\cdot)\right|_{t=t_{p}^{*}}=\frac{1}{1+\left(\left(\lambda_{01} / \lambda_{12}\right)^{\lambda_{12} /\left(\lambda_{01}-\lambda_{12}\right)-\lambda_{01} /\left(\lambda_{01}-\lambda_{12}\right)}\right)}, \\
& \left.h_{1}^{\mathrm{opt}}(\cdot)\right|_{t=t_{p}^{*}}=\frac{1}{1+\left(\lambda_{01} / \lambda_{12}\right)^{-1}}, \\
& \left.h_{1}^{\mathrm{opt}}(\cdot)\right|_{t=t_{p}^{*}}=\frac{1}{1+\left(\lambda_{12} / \lambda_{01}\right)} .
\end{aligned}
$$

This inevitably gives

$$
\left.h_{1}^{\mathrm{opt}}(\cdot)\right|_{t=t_{p}^{*}}=\frac{\lambda_{01}}{\lambda_{01}+\lambda_{12}} .
$$

The parallel to the derivation of (23), (24), (25), (26), (27), (28), (29), (30), (31), (32), (33), (34), and (35) leads to

$$
\left.h_{2}^{\mathrm{opt}}(\cdot)\right|_{t=t_{p}^{*}}=\frac{\lambda_{12}}{\lambda_{01}+\lambda_{12}} .
$$

Now, it raises the problem of the combinational concept of both the multioptional doctrine [2-4] (for the objectively existing phenomena) and the multialternative entropy paradigm $[1,5]$ (for the subjectively preferred choices and decision making).

The part of the general problem dealing with the objectively existing maximum of probability $P_{1}(t)$ is solved with the help of the multioptional doctrine, whereas the options for the part of the material improvement for the diversity of the objective parameters $\lambda_{01}$ and $\lambda_{12}$ is finally going to be made with the use of SEMP.

2.4. Simulation. A numerical case study is proposed in order to illustrate the theoretical concepts and clarify mathematical derivations of (1), (2), (3), (4), (5), (6), (7), (8), (9), (10), (11), (12), (13), (14), (15), (16), (17), (18), (20), (21), (22), (23), (24), (25), (26), (27), (28), (29), (30), (31), (32), (33), (34), (35), and (36). The conducted simulation detailing the case study performs the role of an aid for the possible applications of the multioptional doctrine.

The usefulness of this methodology depends upon the individual subjective preferences of the persons conducting the experimentation procedure, and details may vary in instruments, materials, sets or sequences, and so on.

Also, for example, for data collection, the simplest forms may be used, like basic spreadsheets or something similar. It is not so important for the given stage of the developed theoretical approach.

The purpose of the modeling carried out here is to manifest the damages presented herein which were due to the failures of the ability of theoretical concepts to be applied to a wide range of material-degrading phenomena.

For the computer simulation in the framework of the approaches of (1), (2), (3), (4), (5), (6), (7), (8), (9), (10), (11), (12), (13), (14), (15), (16), (17), (18), (20), (21), (22), (23), (24), (25), (26), (27), (28), (29), (30), (31), (32), (33), (34), (35), and (36), there have been calculation data used which are visible in the diagrams plotted in Figures $2-4$.

Figure 2 shows the curves of state " 1 " (see Figure 1) probability $P_{1}(t) . P_{101}\left(\lambda_{01}, 1 \cdot 10^{-3}\right)$ stands for $P_{1}(t)$ maximums in the case of the transition intensity $\lambda_{01}$ variation and $\lambda_{12}=1 \cdot 10^{-3}$ plotted as the function of $T_{01}\left(\lambda_{01}, 1\right.$. $10^{-3}$ ) when the objective parameters $\lambda_{01}$ and $\lambda_{12}$ have corresponding values. $T_{01}\left(\lambda_{01}, 1 \cdot 10^{-3}\right)$ was computed with help of (12). $P_{112}\left(5 \cdot 10^{-3}, \lambda_{12}\right)$ stands for $P_{1}(t)$ maximums in the case of the transition intensity $\lambda_{12}$ variation and $\lambda_{01}=5 \cdot 10^{-3} \cdot P_{112}\left(5 \cdot 10^{-3}, \lambda_{12}\right)$ was plotted as the function of $T_{12}\left(5 \cdot 10^{-3}, \lambda_{12}\right)$ similar to the method of a phase portrait $\left(P_{101}\left(\lambda_{01}, 1 \cdot 10^{-3}\right)-T_{01}\left(\lambda_{01}, 1 \cdot 10^{-3}\right)\right)$. The next seven curves of $P_{1}(t), P_{1}\left(0.6 \cdot 10^{-3} \ldots 4 \cdot 10^{-3}, 1 \cdot 10^{-3}, t\right)$, indicate the situation of the transition intensity $\lambda_{01}$ variation and $\lambda_{12}=1 \cdot 10^{-3}$ with the corresponding maximum values crossed with the $P_{101}\left(\lambda_{01}, 1 \cdot 10^{-3}\right)$ line. The last seven curves 


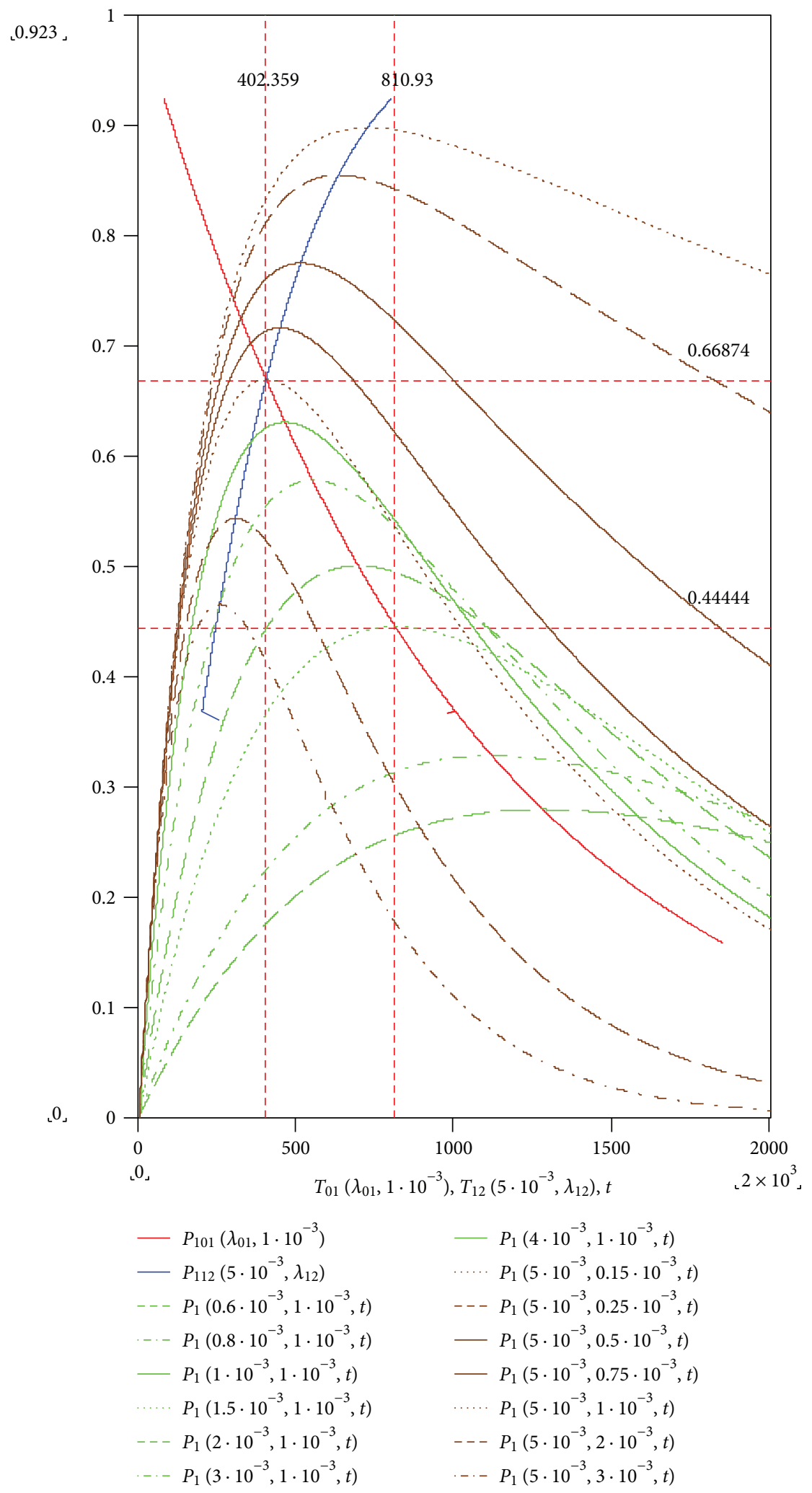

FIGURE 2: Divergence of the objectively existing maximum for the probability of the materials damaged; however, there is no fractured state in the case of the transition intensity variations.

of the probability $P_{1}(t), P_{1}\left(5 \cdot 10^{-3}, 0.15 \cdot 10^{-3} \ldots 3 \cdot 10^{-3}, t\right)$, are plotted for the other alternative case of the transition intensity $\lambda_{12}$ variation and $\lambda_{01}=5 \cdot 10^{-3}$.
Also, there are two points with the coordinates of $(402.359,0.66874)$ and $(810.93,0.44444)$ denoted for the specified examples in Figure 2. 


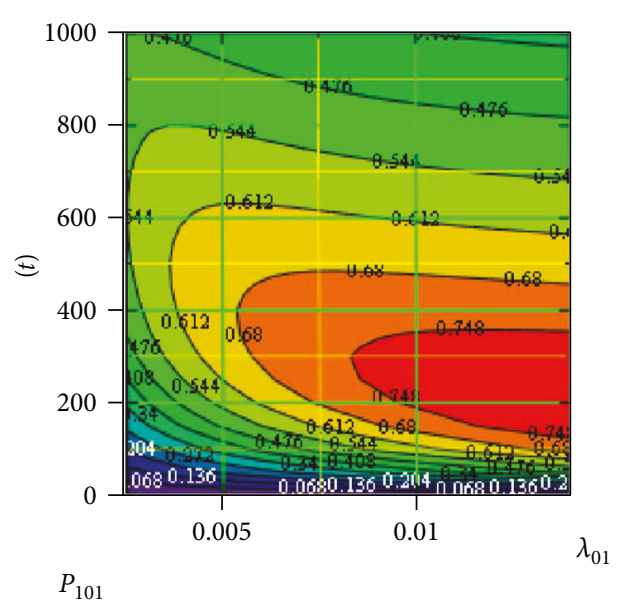

FIGURE 3: Change of the probability of the materials possible and assumed by the damaged state of the experimental data. However, in the illustrated diagram the materials are not fractured. By supposition, the represented change occurs with the variation of the transition intensity from the normal state, with the transition intensity to the fractured state being steady.

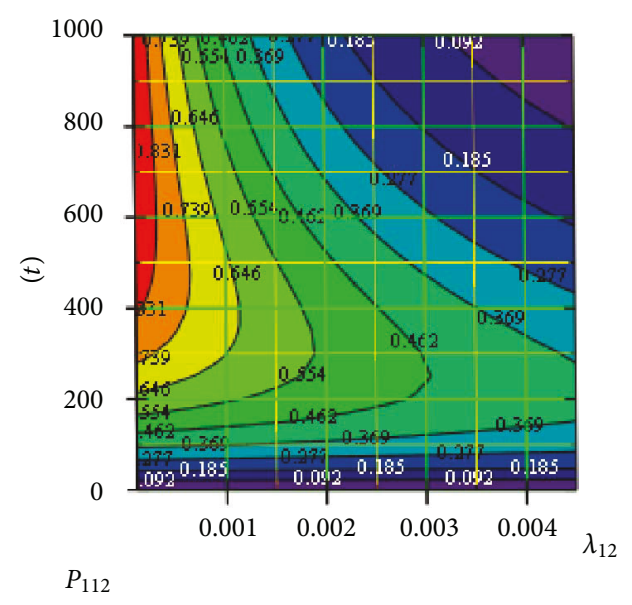

Figure 4: Change of the probability of the materials possible and assumed by the damaged state of experimental data in time. However, in the illustrated diagram the materials are not fractured. By supposition, the represented change occurs with the variation of the transition intensity from the damaged state, with the transition intensity from the normal state being steady.

The results of numerical modeling shown in Figure 3 represent a contour plot for the probability $P_{1}(t)$ of the damaged but not ruined state " 1 " denoted as $P_{101}$ and correspond to the situation described above.

Figure 4 illustrates the situation when $P_{1}(t)$ changes in time as a result of the transition intensity $\lambda_{12}$ variation and $\lambda_{01}=5 \cdot 10^{-3}$.

\section{Results and Discussion}

3.1. Analysis of the Obtained Results. The obtained results represented by the diagrams shown in Figures 2-4 brightly distinguish the two major options for the improvement of the material described above, increasing its reliability measures: the required change of the transition intensities $\lambda_{12}$ and $\lambda_{01}$. If the case is not so simple, certain additional assumptions can be made.

3.2. Discussion. The demonstrated hereinafter possibility of the multioptional optimality doctrine application in the areas of exclusively objective options in contrast to that which is subjectively preferred by somebody's alternatives gives the same result as that one found on the basis of the probabilistic approach [2-4]. This is an undoubtedly fragmented piece of evidence proving the substantiation of the proposed doctrine.

The optimal maintenance periodicity can be predetermined not necessarily with the probability $P_{1}(t)$ maximum value. In such a case, a more complicated approach perhaps should be used; however, the application of SEMP still looks even more attractive since it implies taking into account someone's individual subjective preference distribution. Therefore, the role of the theoretical evolutions as well as subjective analysis $[1,5]$ is apparently going to be increased.

With regard to material science and its theory and applications traced with the references of [6-12], the presented herewith entropy approach also gives some plausible results.

\section{Conclusions}

The case study, analyzed in this paper on aeronautical and aerospace materials and structural damages due to the failures of theoretical concepts, proves that the described doctrine allows obtaining the objectively existing optimal values not with the help of probabilistic but rather with multioptimal hybrid effectiveness functions.

Conditions of uncertainty embodied in the corresponding entropies are crucial to the two following aspects: objective hybrid optional and subjective preference function optimal distributions.

The preference functions give the possibility of the considered alternative material assessment with respect to the preference entropy uncertainty measure.

In further research, some other effectiveness functions and their variables should be considered. Also, it would be useful to find more theoretical results and applicable areas of the hybrid optional optimality doctrine as well as the theory of subjective analysis.

\section{Conflicts of Interest}

The author declares that there is no conflict of interests regarding the publication of this paper.

\section{References}

[1] V. Kasianov, Subjective Entropy of Preferences. Subjective Analysis: Monograph, Institute of Aviation, Warsaw, Poland, 2013, http://kasianovv.wixsite.com/entropyofpreferences.

[2] A. V. Goncharenko, "Optimal managerial and control values for active operation," Electronics and control systems, vol. 3, no. 49 , pp. 112-115, 2016.

[3] A. V. Goncharenko, "Optimal UAV maintenance periodicity obtained on the multi-optional basis," in 2017 IEEE 4th 
International Conference Actual Problems of Unmanned Aerial Vehicles Developments (APUAVD), pp. 65-68, IEEE, Kyiv, Ukraine, October 2017.

[4] A. V. Goncharenko, "A hybrid approach to the optimal aeronautical engineering maintenance periodicity determination," Proceedings of the National Aviation University, vol. 72, no. 3, pp. 42-47, 2017.

[5] A. V. Goncharenko, "Aircraft operation depending upon the uncertainty of maintenance alternatives," Aviation, vol. 21, no. 4, pp. 126-131, 2017.

[6] S. M. O. Tavares, S. J. Moreira, P. M. S. T. de Castro, and P. V. Gamboa, "Morphing aeronautical structures: a review focused on UAVs and durability assessment," in 2017 IEEE 4th International Conference Actual Problems of Unmanned Aerial Vehicles Developments (APUAVD), pp. 49-52, IEEE, Kyiv, Ukraine, October 2017.

[7] S. J. Moreira, S. M. O. Tavares, and P. M. S. T. de Castro, "Morphing structures and fatigue: the case of an unmanned aerial vehicle wing leading edge," Fatigue \& Fracture of Engineering Materials \& Structures, vol. 40, no. 10, pp. 16011611, 2017.

[8] R. G. Citarella, P. Carlone, M. Lepore, and G. S. Palazzo, “A FEM-DBEM investigation of the influence of process parameters on crack growth in aluminum friction stir welded butt joints," Key Engineering Materials, vol. 554-557, pp. 21182126, 2013.

[9] O. Fergani, A. Bratli Wold, F. Berto, V. Brotan, and M. Bambach, "Study of the effect of heat treatment on fatigue crack growth behaviour of $316 \mathrm{~L}$ stainless steel produced by selective laser melting," Fatigue \& Fracture of Engineering Materials \& Structures, vol. 41, no. 5, pp. 1102-1119, 2018.

[10] F. Berto and P. Lazzarin, "A review of the volume-based strain energy density approach applied to V-notches and welded structures," Theoretical and Applied Fracture Mechanics, vol. 52, no. 3, pp. 183-194, 2009.

[11] A. Tugarinov, E. Yurchenko, A. Pogrebniak, and M. Regulski, "Calculation method of aerotechnics products fatigue strength subject to cyclic loading," Proceedings of the National Aviation University, vol. 61, no. 4, pp. 111-116, 2014.

[12] A. I. Bogdanovych, "Estimation of kinetic characteristics and modification activation energy of "WX15" in aviation fuel "TC-1"," in Materials of the Sixth World Congress "Aviation in the XXI-st Century", vol. 3, pp. 1.6.9-1.6.13, Kyiv, Ukraine, September 2014. 


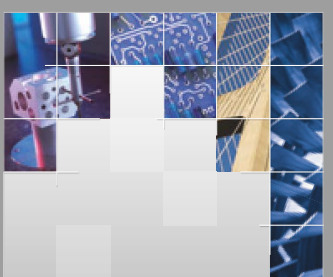

\section{Enfincering}
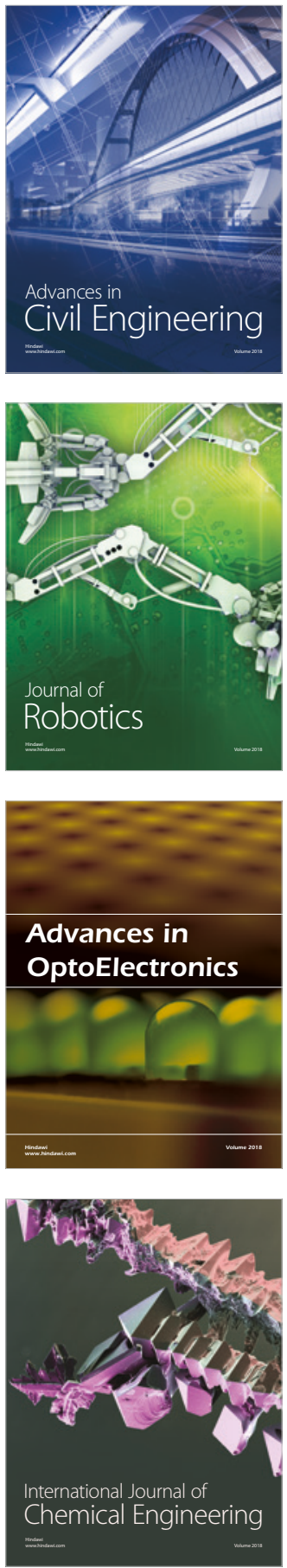

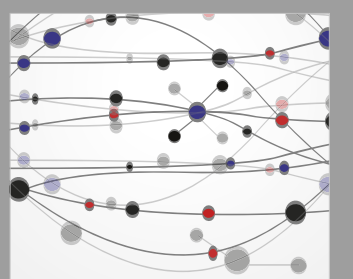

\section{Rotating \\ Machinery}

The Scientific World Journal

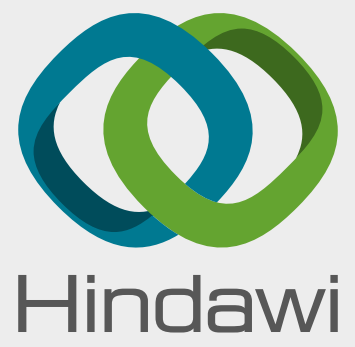

Submit your manuscripts at

www.hindawi.com
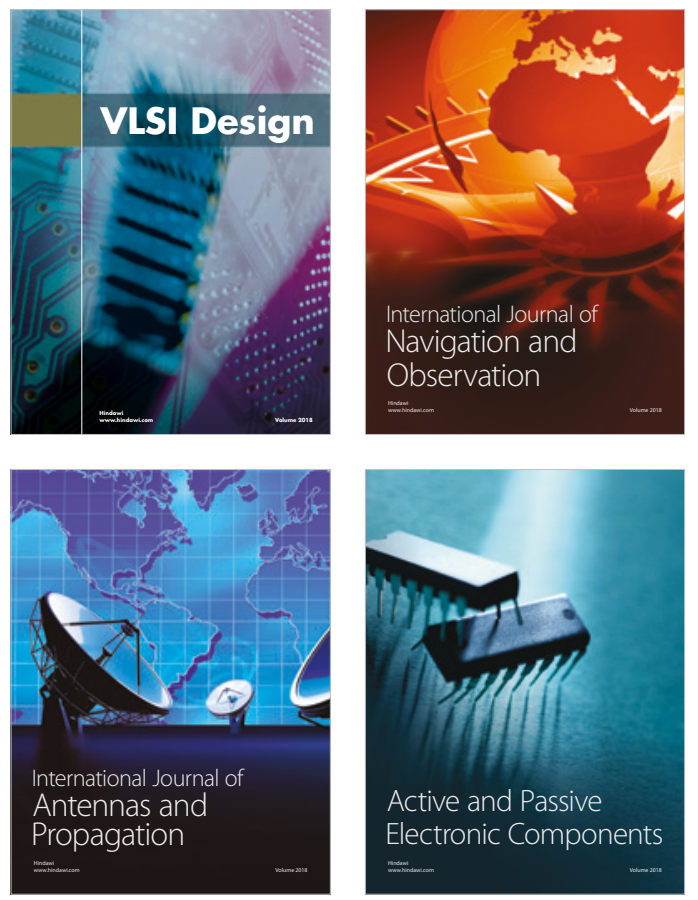
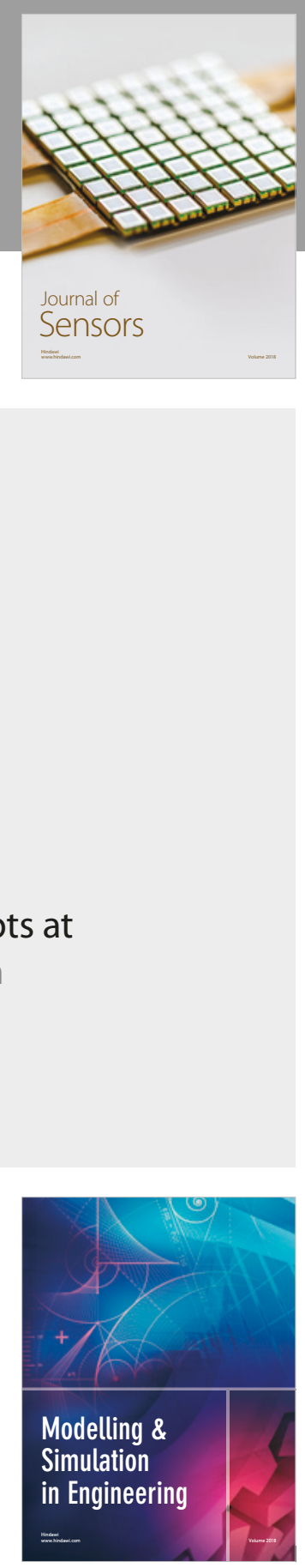

\section{Advances \\ Multimedia}
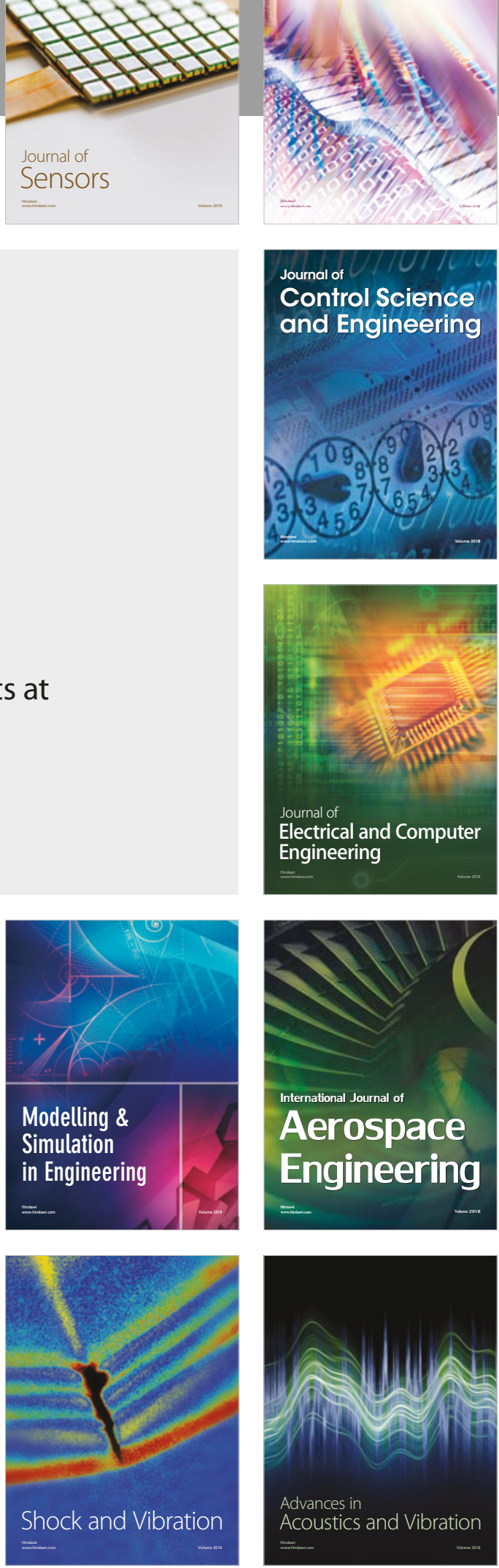